Ann. Biol. anim. Bioch. Biophys., 1977, 17 (3 A), 341-344

\title{
Cathétérisation artérielle chronique du fœtus bovin.
}

\author{
par C. DARDILLAT, J. LEFAIVRE *, J. P. BARLET
}

Station de Physiopathologie de la Nutrition

* Station de Recherches sur l'Elevage des Ruminants

I.N.R.A., Theix, Saint-Genes-Champanelle, 63110 Beaumonf

Summary. Long-term catheterization of a bovine fefus arfery.

A method for catheterizing a tributary of the bovine fetus umbilical vessel is described.

The uterus is incised close to a cotyledon near the uterine artery, thus permitting exteriorization of the fetal membranes. A branch of the umbilical artery is located near a placentome and prepared for catheterization without opening the allantoic cavity. Another cannula is placed in the uterine artery.

The fefal and maternal blood of 5 Jersey cows is thus sampled daily until calving at term : 5, 8, 9, 19, 39 days after surgery. Six calves, including two twins, are born in good health.

This operation is short ( 2 hours) and avoids the risks of infection, so frequent with these kinds of operations. There is no risk of fetal hemorrage during surgery or at calving.

This method is used for the study of fetal calcemia regulation. In the cow carrying twins, we observe spontaneous hypocalcemia and the effect of subsequent calcium infusion.

La cathétérisation chronique des vaisseaux sanguins du fœtus bovin a rarement été effectuée. Hunter et al. (1974) implantent des cathéters dans la veine cave postérieure ; Comline et al. (1974), après avoir utilisé la veine médiane métatarsienne, préfèrent intervenir au niveau d'une branche des vaisseaux ombilicaux. Mais ces auteurs donnent peu de précision sur la technique utilisée et sur la durée pendant laquelle les cathéters sont fonctionnels.

Dans le but d'obtenir des prélèvements sanguins répétés sur des fœutus bovins pour l'étude de la régulation endocrinienne de la calcémie fœtale, nous avons choisi de poser un cathéter sur une artère d'une houppe choriale. Cette technique évite l'ouverture du compartiment amniotique, ce qui exclut d'éventuelles pertes liquidiennes, el diminue les risques de contamination microbienne du fœetus ou des annexes lors de l'opération.

\section{Technique opératoire.}

Nous avons opéré 5 vaches de race Jersiaise, âgées de 3, 6, 7, 8 et 14 ans, pesant $363 \pm 14 \mathrm{~kg}$ au $9 \mathrm{e}$ mois de gestation ef choisies pour leur prédisposition à présenter des hypocalcémies. 
Après un jeûne de 24 h, l'animal est fixé flanc droit contre la table d'opération, postérieurs groupés, allongés vers l'arrière. L'anesthésie induite au masque (fluothane 4 p. 100 dans l'oxygène), est ensuite entretenue à la demande après la pose d'une sonde trachéale à double ballonnet.

La laparotomie se fait sur une longueur de $30 \mathrm{~cm}$, selon une ligne joignant le pli du grassef à l'émergence de la veine mammaire gauche et à partir de celle-ci. La corne utérine gauche esł extériorisée, l'ovaire doit apparaître dans l'angle supéro-postérieur de la plaie.

A environ $30 \mathrm{~cm}$ de l'extrémité de la corne et à $20 \mathrm{~cm}$ de la petife courbure, un gros cotylédon est repéré par palpation, et une boutonnière effectuée le plus près possible de celui-ci en évitant les vaisseaux. La muqueuse utérine doit être incisée. Les membranes fœtales font alors hernie dans la plaie. L'index est introduit entre l'utérus et les enveloppes en direction de la petite courbure, ce qui permet la pose de deux pinces intestinales sur la paroi utérine. L'utérus est incisé entre les deux pinces. Le cotylédon est saisi à travers les membranes et retourné de façon qu'il fasse hernie dans la plaie. Le faisceau vasculaire, sortant du cotylédon, est alors nettement visible. L'artère est repérée, disséquée sur $3 \mathrm{~cm}$, ligaturée à son entrée dans le cotylédon. Une tresse ombilicale est glissée sous l'artère et servira de clamp. L'artère est à moitié incisée au ciseau, perpendiculairement à son axe ef un cathéter $\left(^{1}\right)$ est introduit par l'orifice sur $2 \mathrm{~cm}$, jusqu'à une bague $\left({ }^{2}\right)$, asujettie préalablement sur le cathéter. Celui-ci est solidement fixé par la tresse ombilicale maintenue serrée sur le vaisseau par une ligature à la soie 3. Une deuxième ligature prise derrière la bague et autour du vaisseau complète la fixation. L'utérus est alors refermé par un surjet enfouissant, en reculant progressivement les pinces qui assuraient l'hémostase. Le cathéter sort par la plaie et, après une portion libre d'environ $60 \mathrm{~cm}$ dans la cavité péritonéale, est glissé en sous-cutané sur $40 \mathrm{~cm}$. Il est ensuite extériorisé par une contre-ouverfure sur le flanc gauche.

A l'occasion de cette opération, sur 3 animaux, un cathéter semblable a été posé sur une artère de l'utérus et extériorisé comme précédemment. La plaie abdominale est suturée plan par plan.

Une perfusion de 1 I de sérum glucosé est effectuée à la fin de l'opération et 4 millions d'Ul de pénicilline el $4 \mathrm{~g}$ de streptomycine sont injectés par voie intramusculaire.

Les prélèvements de $5 \mathrm{ml}$ de sang sont effectués deux fois par jour avec le maximum de précautions d'aseptie. De plus, le cathéter est lavé extérieurement par une solution antiseptique d'éthylmercurythiosalicylate d̀ 1 p. 100 dans l'alcool à $90^{\circ}$ (Mallor et Slater, 1973) et $0,1 \mathrm{ml}$ est injecté dans le cathéter après le rinçage au sérum hépariné.

\section{Résultats et discussion.}

Les 5 opérations effectuées se sont déroulées de façon satisfaisante ef ont duré chacune moins de 2 h $1 / 2$; les vaches étaient debout une demi-heure après l'opération et il n'y a pas eu de suites opératoires. Tous les veaux sont nés à terme 5 à 40 jours

(1) Cathéter en PVC : longueur $1 \mathrm{~m}$, diamètre extérieur $2-2,5 \mathrm{~mm}$; intérieur 1,2 - 1,5 mm.

(2) Bague en PVC : longueur $5 \mathrm{~mm}$, mêmes diamètres que le cathéter. 
après l'opération (tabl. 1). Une vache porteuse de jumeaux a présenté $24 \mathrm{~h}$ après l'opération une hypocalcémie qui a disparu normalement après perfusion de glutamate de calcium. Cet accident pathologique, attendu sur ces animaux prédisposés à cette affection, peut également provenir du choc opératoire qui peut provoquer une décharge d'adrénaline entraînant une hypersécrétion de calcitonine responsable du syndrome.

TABLEAU 1

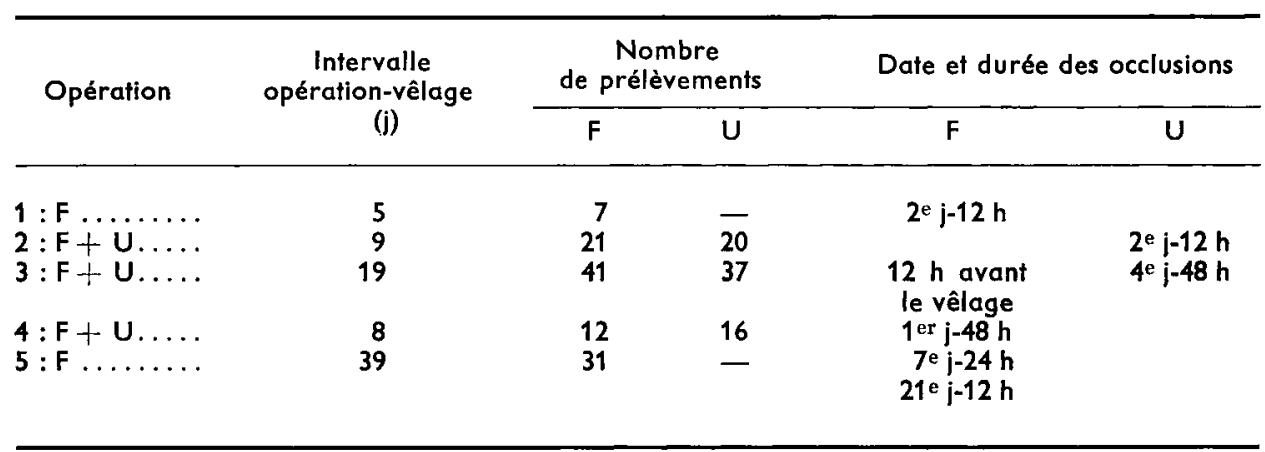

$\mathbf{F}=$ cathéter implanté dans l'artère d'une houppe choriale.

$U=$ cathéter implanté dans une artère utérine.

Le fonctionnement des cathéters fœetaux et maternels a été excellent (tabl. 1) malgré quelques cas passagers d'obstruction. Des occlusions sont souvent observées lorsqu'on utilise des cathéters vasculaires chroniques; la raison la plus courante en est la formation d'un clapet de fibrine à l'extrémité de ceux-ci. Ce phénomène paraît peu probable dans notre cas puisque les occlusions que nous avons observées étaient temporaires; de plus, le sang fotal a un pouvoir coagulant plus faible que celui de l'adulte (Fault et Ward, 1960). Il a souvent été mentionné (Soma, White et Kane, 1971) la production de spasmes sur les vaisseaux ombilicaux conduisant à une occlusion définitive. En fait, nous avons eu une plus grande fréquence d'occlusions temporaires sur les cathéters d'artère utérine que sur les cathéters fœtaux, peuf-être à cause d'une obstruction momentanée du cathéter consécutive à des compressions provoquées par les mouvements du fœtus ou par des contractions utérines.

La pose simultanée d'un cathéter sur une artère utérine et sur celle d'une houppe choriale constitue déjà un modèle expérimental satisfaisant pour l'étude des échanges materno-fœetaux transplacentaires (Barlet et al., 1976). Cette technique présente de nombreux avantages : elle est rapide $(2 \mathrm{~h})$, ne comporte pas de risques de pertes de liquide amniotique ni de risques hémorragiques fœetaux dangereux. Elle conserve l'intégrité des vaisseaux du fœus ef limite les risques d'infection au minimum. Enfin, une surveillance du vêlage n'est pas nécessaire étant donné l'absence d'hémorragie consécutive à l'arrachage du cathéter.

Accepté en décembre 1976.

Remerciements. - Ce travail a pu être réalisé grâce à l'ATP nº A 655-1310 du Centre National de la Recherche Scientifique. 


\section{Références}

BARLET J. P., GAREL J. M., LefaIVRE J., DARDILLAT C., 1976. Régulation endocrinienne de la calcémie fœetale chez la vache ef la brebis. J. Physiol., 72, 34 A.

COMLINE R. S., HALL L. W., LAVELLE R. B., NATHANIELSZ P. M., SILVER M., 1974. Parturition in the cow : endocrine changes in animals with chronically implanted catheters in the fotal and maternal circulations. J. Endocr., 63, 451-472.

FAUTL P., WARD H. A., 1960. Clotting activity of maternal and foetal sheep blood. J. Physiol. (Lond.), 150, 607.

HUNTER J. T., WELCH R. A. S., FAIRCLOUGH R. J., HEATHER BARR, SEAMARK R. J., 1974. Fetal cestrogens and maternal progestins when the bovine foetus was infused with dexamethasone. J. Reprod. Fert., 36, 469-470.

MELLOR D. J., SLATER J. S., 1973. The use of chronic catheterization fechniques in foetal sheep. Br. Vet. J., 129, 260-266.

SOMA L. R., WHITE R. J., KANE P. B., 1971. Surgical preparation of a chronic maternal foetal model in pregnant sheep : a technique for the measurement of middle uterine blood flow, umbilical blood flow and foetal sampling in the awake sheep. J. Surg. Res., 11, 85. 